\title{
TITULO DEL LIBRO: HYPERTHINKING. CREATING A NEW MINDSET FOR THE AGE OF NETWORKS
}

EDITORIAL: GOWER PUBLISHING, LTD.

FECHA DE PUBLICACIÓN: 2012

AUTOR: PHILIP WEISS

NÚMERO DE PÁGINAS: 186

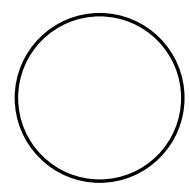

JANNET CANO

En esta obra, el autor parte de la necesidad de adaptar la forma en que pensamos a la turbulencia económica y política, propia de los tiempos en que vivimos. Es en tal contexto que propone cuatro principios - hypershift, hyperlearn, hyperlink e hyperact- que facilitan el desarrollo de una actitud mental más fluida que incorpora el cambio, la creatividad y la tecnología. Esta nueva actitud mental nos permitirá realizar una mejor labor de liderazgo y de gestión.

El primer principio, hypershift, se refiere a la importancia de entender nuestros paradigmas existentes y de aprender a modificar nuestras percepciones.

El segundo, hyperlearn, combina el aprendizaje continuo y la experimentación, para afinar nuestras habilidades mentales.

El tercero de ellos, hyperlink, está enfocado en el imperativo de utilizar las nuevas tecnologías y de aplicarlas en cada aspecto de nuestras vidas aprovechando el potencial de las redes sociales.

Finalmente, el cuarto principio, hyperact, resalta la trascendencia de hacer y los retos que se enfrentan cuando se proponen nuevas ideas con soporte tecnológico. Tiene que ver con la habilidad de concebir y de probar ideas para determinar su utilidad.

El reto que plantea el autor es que nos convirtamos en hyperthinkers, para que, aplicando los cuatro principios en el día a día, cambiemos al mundo adaptando nuestra forma de pensar individual a las nuevas realidades de estos tiempos. 